\title{
$(\ell, 0)$-Carter Partitions and their crystal theoretic interpretation
}

\author{
Chris Berg ${ }^{\dagger}$ and Monica Vazirani ${ }^{\ddagger}$ \\ Department of Mathematics, UC Davis, USA, 95616
}

\begin{abstract}
In this paper we give an alternate combinatorial description of the " $(\ell, 0)$-Carter partitions". Our main theorem is the equivalence of our combinatoric and the one introduced by James and Mathas (A q-analogue of the Jantzen-Schaper theorem). The condition of being an $(\ell, 0)$-Carter partition is fundamentally related to the hook lengths of the partition. The representation-theoretic significance of their combinatoric on an $\ell$-regular partition is that it indicates the irreducibility of the corresponding Specht module over the finite Hecke algebra. We use our result to find a generating series which counts the number of such partitions, with respect to the statistic of a partition's first part. We then apply our description of these partitions to the crystal graph $B\left(\Lambda_{0}\right)$ of the basic representation of $\widehat{\mathfrak{s l}_{\ell}}$, whose nodes are labeled by $\ell$-regular partitions. Here we give a fairly simple crystal-theoretic rule which generates all $(\ell, 0)$-Carter partitions in the graph of $B\left(\Lambda_{0}\right)$.

Résumé. Dans cet article, nous donnons une description combinatoire alternative des partitions $(\ell, 0)$-Carter. Notre théorème principal est une équivalence entre notre combinatoire et celle introduite par James et Mathas (A q-analogue of the Jantzen-Schaper theorem). La propriété ( $\ell, 0)$-Carter est fondamentalement liée aux longueurs des équerres de la partition. En terme de théorie des représentations, leur combinatoire pour une partition $\ell$-réguliére permet de déterminer l'irréducibilité du module de Specht spécialisé sur l'algèbre de Hecke finie. Nous utilisons notre résultat pour déterminer leur série génératrice en fonction de la taille de la première part. Nous utilisons ensuite notre description de ces partitions au graphe cristallin $B\left(\Lambda_{0}\right)$ de la représentation basique de $\widehat{\mathfrak{s l}}_{\ell}$, dont les noeuds sont étiquetés par les partitions $\ell$-régulières. Nous donnons un règle cristalline relativement simple permettant d'engendrer toutes les partitions $\ell$-régulières $(\ell, 0)$-Carter dans le graphe de $B\left(\Lambda_{0}\right)$.
\end{abstract}

Keywords: Representation theory, Hecke algebras, Combinatorics of Young tableaux, Crystals of affine Lie algebras

\section{Introduction}

\subsection{Preliminaries}

Let $\lambda$ be a partition of $n$ and $\ell \geq 2$ be an integer. We will use the convention $(x, y)$ to denote the box which sits in the $x^{\text {th }}$ row and the $y^{\text {th }}$ column of the Young diagram of $\lambda$. Throughout this paper, all of our partitions are drawn in English notation. $\mathcal{P}$ will denote the set of all partitions. An $\ell$-regular partition is

\footnotetext{
${ }^{\dagger}$ Partially supported by the National Science Foundation under Grant No DMS-0135345

${ }^{\ddagger}$ Supported in part by NSF grant DMS-0301320

1365-8050 (C) 2008 Discrete Mathematics and Theoretical Computer Science (DMTCS), Nancy, France
} 
one in which no part occurs $\ell$ or more times. The length of a partition $\lambda$ is defined to be the number of nonzero parts of $\lambda$ and will be denoted len $(\lambda)$.

The hook length of the $(a, c)$ box of $\lambda$ is defined to be the number of boxes to the right and below the box $(a, c)$, including the box $(a, c)$ itself. It will be denoted $h_{(a, c)}^{\lambda}$.

A removable $\ell$-rim hook in $\lambda$ (also called an $\ell$-ribbon of $\lambda$ ) is a connected sequence of $\ell$ boxes in the Young diagram of $\lambda$, containing no $2 \times 2$ square, such that when removed from $\lambda$, the remaining diagram is the Young diagram of some other partition. We will often abbreviate and call a removable $\ell$-rim hook an $\ell$-rim hook.

Any partition which has no removable $\ell$-rim hooks is called an $\ell$-core. Every partition $\lambda$ has a well defined $\ell$-core, which is obtained by removing $\ell$-rim hooks from the outer edge while at each step the removal of a hook is still a (non-skew) partition. The $\ell$-core is uniquely determined from the partition, independently of choice of the order in which one successively removes $\ell$-rim hooks. The set of all $\ell$-cores will be denoted $\mathcal{C}_{\ell}$. See [4] for more details.

Removable $\ell$-rim hooks which are flat (i.e. those whose boxes all sit in one row) will be called horizontal $\ell$-rim hooks. These are also commonly called $\ell$-ribbons with spin 0 . Removable $\ell$-rim hooks which are not flat will be called non-horizontal $\ell$-rim hooks.

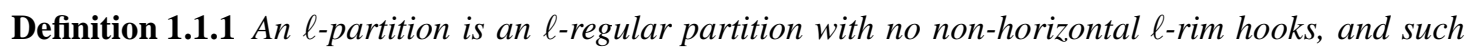
that after removing any number of horizontal $\ell$-rim hooks, the remaining diagram has no non-horizontal

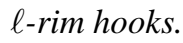

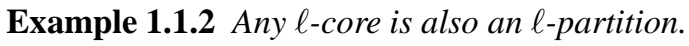

Example 1.1.3 $(6,4)$ is a 3-partition, as well as a $\ell$-partition for $\ell>7$ but is not a 2, 4, 5, 6, 7-partition. It is an $\ell$-core for $\ell>7$.

Definition 1.1.4 For a fixed field $\mathbb{F}$ and $0 \neq q \in \mathbb{F}$, the finite Hecke Algebra $H_{n}(q)$ is defined to be the algebra over $\mathbb{F}$ generated by $T_{1}, \ldots, T_{n-1}$ with relations

$$
\begin{array}{ll}
T_{i} T_{j}=T_{j} T_{i} & \text { for }|i-j|>1 \\
T_{i} T_{i+1} T_{i}=T_{i+1} T_{i} T_{i+1} & \text { for } i<n-1 \\
T_{i}^{2}=(q-1) T_{i}+q & \text { for } i \leq n-1 .
\end{array}
$$

In this paper we will always assume that $q \neq 1$, that $q \in \mathbb{F}$ is a primitive $\ell$ th root of unity (so necessarily $\ell \geq 2$ ) and that the characteristic of $\mathbb{F}$ is zero.

Similar to the symmetric group, a construction of the Specht module $S^{\lambda}=S^{\lambda}[q]$ exists for $H_{n}(q)$ (see [1]). For $k \in \mathbb{Z}$ let

$$
\nu_{\ell}(k)= \begin{cases}1 & \ell \mid k \\ 0 & \ell \chi k\end{cases}
$$

It is known that the Specht module $S^{\lambda}$ indexed by an $\ell$-regular partition $\lambda$ is irreducible (over a field of characteristic zero) if and only if

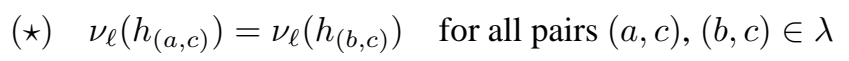

(see [5]). $\ell$-regular partitions which satisfy $(\star)$ have been called in the literature $(\ell, 0)$-Carter partitions. So, an equivalent condition for the irreducibility of the Specht module indexed by an $\ell$-regular partition 
is that the hook lengths in a column of the partition $\lambda$ are either all divisible by $\ell$ or none of them are, for every column in $\lambda$ (see [2] for general partitions, when $\ell \geq 3$ ).

All of the irreducible representations of $H_{n}(q)$ have been constructed abstractly when $q$ is a primitive $\ell$ th root of unity. These modules are indexed by $\ell$-regular partitions $\lambda$, and are called $D^{\lambda} . D^{\lambda}$ is the unique simple quotient of $S^{\lambda}$ (see [1] for more details). In particular $D^{\lambda}=S^{\lambda}$ if and only if $S^{\lambda}$ is irreducible and $\lambda$ is $\ell$-regular.

\subsection{Main results}

Here we summarize the main results of this paper. Section 2 shows the equivalence of $\ell$-partitions and $(\ell, 0)$-Carter partitions (see Theorem 2.1.6). Section 3 gives a different classification of $\ell$-partitions which allows us to give an explicit formula for a generating function for the number of $\ell$-partitions with respect to the statistic of a partitions first part. Finally, in Section 4 we give a crystal theoretic interpretation of $\ell$-partitions. There we explain where in the crystal graph $B\left(\Lambda_{0}\right)$ one can expect to find $\ell$-partitions (see Theorems 4.3.1, 4.3.2 and 4.3.3).

\section{2 -partitions}

We now claim that an $\ell$-regular partition is an $\ell$-partition if and only if it satisfies $(\star)$ (We drop the $(\ell, 0)$ Carter partition notation and just use the combinatorial condition of $(\star))$. To prove this, we will first need two lemmas which tell us when we can add/remove horizontal $\ell$-rim hooks to/from a diagram.

\subsection{Equivalence of the combinatorics}

We omit the proofs of several lemmas in the interest of space.

Lemma 2.1.1 For $\lambda$ a partition which does not satisfy $(\star)$, if we add a horizontal $\ell$-rim hook to $\lambda$ to form a new partition $\mu, \mu$ will also not satisfy $(\star)$.

Example 2.1.2 Let $\lambda=(14,9,5,2,1)$ and $\ell=3$. This partition does not satisfy $(\star)$. For instance, $3 \mid h_{(3,3)}^{\lambda}=3$ but $3 \backslash h_{(2,3)}^{\lambda}=8$. Let $\lambda[i]$ denote the partition obtained when adding a horizontal $\ell$-rim hook to the ith row of $\lambda$ (when it is still a partition). Adding a horizontal 3-rim hook in row 1 will not change $h_{(2,3)}^{\lambda}$ or $h_{(3,3)}^{\lambda}$. Adding a horizontal 3 -rim hook to row 2 will change $h_{(2,3)}^{\lambda[2]}$ to 11 , which is equal to $h_{(2,3)}^{\lambda}$ mod 3. Adding a horizontal 3 -rim hook to row 4 will change $h_{(2,3)}^{\lambda}$ and $h_{(3,3)}^{\lambda}$, but one column to the right, we see that now $h_{(2,4)}^{\lambda[4]}=8$ and $h_{(3,4)}^{\lambda[4]}=3$. These sorts of observations hold in general and can be used to prove Lemma 2.1.1.

Lemma 2.1.3 Suppose $\lambda$ does not satisfy $(\star)$. Suppose $a<b$ and suppose that $\ell$ divides exactly one of $h_{(a, c)}^{\lambda}$ and $h_{(b, c)}^{\lambda}$. Let $\nu$ be $\lambda$ with a removed horizontal $\ell$-rim hook (if a horizontal $\ell$-rim hook exists). Then, as long as $\nu$ still has the box $(b, c), \nu$ does not satisfy $(\star)$.

Remark 2.1.4 Lemmas 2.1.1 and 2.1.3 can actually be strengthened to show that when removing or adding a horizontal $\ell$-rim hook to a partition which does not satisfy $(\star)$, the violation to $(\star)$ occurs in the same rows as the original partition. This will be useful in the proof of Theorem 2.1.6.

Example 2.1.5 $(5,4,1)$ does not satisfy $(\star)$ for $\ell=3$. The boxes $(1,2)$ and $(2,2)$ are a violation of $(\star)$. Removing a horizontal 3 -rim hook will give the partition $(5,1,1)$ which does satisfy $(\star)$. Note that this does not violate Lemma 2.1.3, since the removed horizontal 3-rim hook contains the box $(2,2)$. 
Theorem 2.1.6 A partition is an $\ell-p a r t i t i o n$ if and only if it satisfies $(\star)$.

Proof: Let $\lambda$ be a partition.

$\Leftarrow$ Suppose $\lambda$ is not an $\ell$-partition. If $\lambda$ is not $\ell$-regular then let $i$ be given so that $\lambda_{i}=\lambda_{i+1}=\cdots=$ $\lambda_{i+\ell-1} \neq 0$. Then exactly one of $h_{\left(i, \lambda_{i}\right)}^{\lambda}, h_{\left(i+1, \lambda_{i}\right)}^{\lambda}, \ldots, h_{\left(i+\ell-1, \lambda_{i}\right)}^{\lambda}$ is divisible by $\ell$. Therefore, $\lambda$ does not satisfy $(\star)$.

Otherwise, suppose $\lambda$ is $\ell$-regular and a non-horizontal $\ell$-rim hook can be removed from $\lambda$, with upper rightmost box $(a, c)$ and lower leftmost box $(b, d)$ with $a<b$ and $c \geq d$. Then $h_{(a, d)}^{\lambda}=\ell$ and $h_{(b, d)}^{\lambda}<\ell$ so $\lambda$ does not satisfy $(\star)$. If no non-horizontal $\ell$-rim hooks appear immediately in the partition, then remove horizontal $\ell$-rim hooks from $\lambda$ until you obtain a partition $\mu$ with a non-horizontal $\ell$-rim hook. Then by the same argument, $\mu$ does not satisfy ( $\star$ ). Now add back to $\mu$ the horizontal $\ell$-rim hooks which were removed from $\lambda$. By Lemma 2.1.1, each time a horizontal $\ell$-rim hook is added to $\mu$ the resulting partition will not satisfy $(\star)$. Thus $\lambda$ does not satisfy $(\star)$.

$\Rightarrow$ Suppose $\lambda$ is an $\ell$-partition and that $\lambda$ does not satisfy $(\star)$. We will suppose that there exists boxes $(a, c)$ and $(b, c)$ in $\lambda$ so that $\ell$ divides exactly one of $h_{(a, c)}^{\lambda}$ and $h_{(b, c)}^{\lambda}$.

Case 1: Suppose that $a<b$ and that $\ell \mid h_{(a, c)}^{\lambda}$. Then without loss of generality we may assume that $b=a+1$. We leave to the interested reader the proof of the fact that if $\ell \mid h_{(a, c)}^{\lambda}$ then there exists at least one removable $\ell$-rim hook in the Young diagram for $\lambda$ (see [4]). By assumption it must be horizontal. If one exists which does not contain the box $(b, c)$ then remove it and let $\lambda^{(1)}$ be $\lambda$ without this $\ell$-rim hook. By Lemma 2.1.3, as long as we did not remove the $(b, c)$ box, $\lambda^{(1)}$ will still not satisfy $(\star)$. Then there are boxes $\left(a, c_{1}\right)$ and $\left(b, c_{1}\right)$ for which $\ell \mid h_{\left(a, c_{1}\right)}^{\lambda^{(1)}}$ but $\ell \chi h_{\left(b, c_{1}\right)}^{\lambda^{(1)}}$. Remark 2.1.4 above explains why we can assume that the violation to $(\star)$ are in the same rows $a$ and $b$ of $\lambda^{(1)}$. We apply the same process as above repeatedly until we can remove a horizontal $\ell$-rim hook from the partition $\lambda^{(k)}$ which contains the $\left(b, c_{k}\right)$ box (i.e. we are not in the case where we can apply Lemma 2.1.3) and cannot remove a horizontal $\ell$-rim hook from row $a$. Let $d$ be so that $h_{(b, d)}=1$. Such a $d$ must exist since we are removing a horizontal $\ell$-rim hook from this row. Since $\left(b, c_{k}\right)$ is removed from $\lambda^{(k)}$ when we remove the horizontal $\ell$-rim hook, $h_{\left(b, c_{k}\right)}^{\lambda^{(k)}}<\ell\left(\ell\right.$ does not divide $h_{\left(b, c_{k}\right)}^{\lambda^{(k)}}$ by assumption, so in particular $\left.h_{\left(b, c_{k}\right)}^{\lambda^{(k)}} \neq \ell\right)$. Note that $h_{\left(a, c_{k}\right)}^{\lambda^{(k)}}=h_{\left(b, c_{k}\right)}^{\lambda^{(k)}}+h_{(a, d)}^{\lambda^{(k)}}-1, \ell \mid h_{\left(a, c_{k}\right)}^{\lambda^{(k)}}$ and $\ell \chi h_{\left(b, c_{k}\right)}^{\lambda^{(k)}}$, so $\ell \chi\left(h_{(a, d)}^{\lambda^{(k)}}-1\right)$. If $h_{(a, d)}^{\lambda^{(k)}}-1>\ell$ then we could remove a horizontal $\ell$-rim hook from row $a$, which we cannot do by assumption. Otherwise $h_{(a, d)}^{\lambda^{(k)}}<\ell$. Then a non-horizontal $\ell$-rim hook exists starting at the rightmost box of the $a$ th row, going left to $(a, d)$, down to $(b, d)$ and then left until you have covered $\ell$ boxes. This is a contradiction as we have assumed that $\lambda$ was an $\ell$-partition.

Case 2: Suppose that $a<b$ and that $\ell \mid h_{(b, c)}^{\lambda}$. We will reduce this to Case 1 . Without loss of generality we may assume that $b=a+1$ and that $\ell \mid h_{(n, c)}^{\lambda}$ for all $n>a$ (otherwise we are in Case 1). Let $m$ be so that $(m, c) \in \lambda$ but $(m+1, c) \notin \lambda$. Then because $h_{(m, c)}^{\lambda} \geq \ell$, the list $h_{(a, c)}^{\lambda}, h_{(a, c+1)}^{\lambda}=$ $h_{(a, c)}^{\lambda}-1, \ldots, h_{(a, c+\ell-1)}^{\lambda}=h_{(a, c)}^{\lambda}-\ell+1$ consists of $\ell$ consecutive integers. Hence one of them must be divisible by $\ell$. Say it is $h_{(a, c+i)}^{\lambda}$. Also $\ell \times h_{(m, c+i)}^{\lambda}$, since $h_{(m, c+i)}^{\lambda}=h_{(m, c)}^{\lambda}-i$ and $\ell \mid h_{(m, c)}^{\lambda}$. Then we may apply Case 1 to the boxes $(a, c+i)$ and $(m, c+i)$. 
Remark 2.1.7 This result can also be obtained using a more general result of James and Mathas ([5] Theorem 4.20), where they classified which $S^{\lambda}$ remain irreducible for $\lambda \ell$-regular. However, we have included this proof to emphasize the simplicity of the theorem and its simple combinatorial proof in this context.

Remark 2.1.8 When $q$ is a primitive $\ell$ th root of unity, and $\lambda$ is an $\ell$-regular partition, the Specht module $S^{\lambda}$ of $H_{n}(q)$ is irreducible if and only if $\lambda$ is an $\ell$-partition. This follows from what was said above concerning the James and Mathas result on the equivalence of $(\star)$ and irreducibility of Specht modules, and Theorem 2.1.6.

\section{Generating Functions}

Let $\mathcal{L}_{\ell}=\{\lambda \in \mathcal{P}: \lambda$ is an $\ell$-partition $\}$. In this section, we count $\ell$-partitions via generating functions. We study the generating function with the statistic being the first part of the partition. Let $B_{\ell}(x)=$ $\sum_{k=0}^{\infty} b_{k}^{\ell} x^{k}$ where $b_{k}^{\ell}=\#\left\{\lambda: \lambda_{1}=k, \lambda \in \mathcal{L}_{\ell}\right\}$ is the number of $\ell$-partitions with the first part of the partition equal to $k$ (i.e. $B_{\ell}(x)=\sum_{\lambda \in \mathcal{L}_{\ell}} x^{\lambda_{1}}$ ). There is a nice recursion of cores, taking the size of the first part into account. As a result, with respect to this statistic, we get a surprisingly elegant formula for the generating function.

\subsection{Counting $\ell$-cores}

We will count $\ell$-cores first, with respect to the statistic of the first part of the partition. Let

$$
C_{\ell}(x)=\sum_{k=0}^{\infty} c_{k}^{\ell} x^{k}
$$

where $c_{k}^{\ell}=\#\left\{\lambda \in \mathcal{C}_{\ell}: \lambda_{1}=k\right\}$. Note that this does not depend on the size of the partition, only its first part. Also, the empty partition is considered as the unique partition with first part 0 , and is always a core, so that $c_{0}^{\ell}=1$ for every $\ell$.

Example 3.1.1 For $\ell=2$, all 2-cores are staircases, i.e. are of the form $\lambda=(k, k-1, \ldots, 2,1)$. Hence $C_{2}(x)=\sum_{k=0}^{\infty} x^{k}=\frac{1}{1-x}$.

For a partition $\lambda=\left(\lambda_{1}, \ldots, \lambda_{s}\right)$, the $\beta$-numbers $\left(\beta_{1}, \ldots, \beta_{s}\right)$ of $\lambda$ are defined to be the hook lengths of the first column (i.e. $\beta_{i}=h_{(i, 1)}^{\lambda}$ ). Note that this is a simplified version of the $\beta$-numbers defined by James and Kerber in [4], where all definitions in this section can be found. We draw a diagram $\ell$ columns wide with the numbers $\{0,1,2, \ldots, \ell-1\}$ inserted in the first row in order, $\{\ell, \ell+1, \ldots, 2 \ell-1\}$ inserted in the second row in order, etc. Then we circle all of the $\beta$-numbers for $\lambda$. The columns of this diagram are called runners, the circled numbers are called beads, and the diagram is called an abacus. It is well known that a partition $\lambda$ is an $\ell$-core if and only if all of the beads lie in the last $\ell-1$ runners and if any bead is in the abacus, then all of the numbers above it in the same runner also have beads. Recall that $c_{k}^{\ell}=\#\left\{\lambda \in \mathcal{C}_{\ell}: \lambda_{1}=k\right\}$.

Proposition 3.1.2 $c_{k}^{\ell}=\left(\begin{array}{c}k+\ell-2 \\ k\end{array}\right)$. 
Proof: This proof is by induction on $\ell$. For $\ell=2$ this was done in the example above. We next show the relation:

$$
(\sharp) \quad c_{k}^{\ell}=\sum_{j=0}^{k} c_{j}^{\ell-1}
$$

This will prove the result, using induction on all the terms on the right hand side and the fact that $\left(\begin{array}{c}\ell+k-2 \\ k\end{array}\right)=\left(\begin{array}{c}\ell-3 \\ 0\end{array}\right)+\left(\begin{array}{c}\ell-2 \\ 1\end{array}\right)+\cdots+\left(\begin{array}{c}\ell+k-3 \\ k\end{array}\right)$. We give a bijective proof of this relation. Using the abacus description of cores, we describe our bijection as follows:

Given an $\ell$-core with largest part $k$, remove the whole runner which contains the largest bead (the bead with the largest $\beta$-number). The remaining runners can be placed into an $\ell-1$ abacus in order. The remaining abacus will clearly have its first runner empty. This will correspond to an $(\ell-1)$-core with largest part at most $k$. This map gives a bijection between the set of all $\ell$-cores with largest part $k$ and the set of all $(\ell-1)$-cores with largest part at most $k$.

The set of all $(\ell-1)$-cores with largest part at most $k$ has cardinality equal to the right hand side of $(\sharp)$, by induction. Therefore, the set of all $\ell$-cores with largest part $k$ has cardinality $\left(\begin{array}{c}k+\ell-2 \\ k\end{array}\right)$.

Remark 3.1.3 The bijection above between $\ell$-cores with first part $k$ and $(\ell-1)$-cores with first part $\leq k$ has several other beautiful descriptions, using different interpretations of $\ell$-cores. We plan on including these descriptions in a later paper. As far as we know, this bijection is new.

Example 3.1.4 Let $\ell=3$ and $\lambda=(4,2,2,1,1)$. The abacus for $\lambda$ is:

$\begin{array}{ccc}0 & 1 & 2 \\ 3 & 4 & 5 \\ 6 & 7 & 8 \\ 9 & 10 & 11 \\ \vdots & \vdots & \vdots\end{array}$

The largest $\beta$-number is 8 . Removing the whole runner in the same column as the 8 , we get the remaining diagram with runners relabeled for $\ell=2$

$\begin{array}{ccc}0 & 1 & \bigotimes \\ 2 & 3 & \bigotimes \\ 4 & 5 & \bigotimes \\ 6 & 7 & \\ \vdots & \vdots & \end{array}$

These are the $\beta$-numbers for the partition $(2,1)$, which is a 2 -core with largest part $\leq 4$.

From the above reasoning, we obtain $C_{\ell}(x)=\sum_{k \geq 0}\left(\begin{array}{c}k+\ell-2 \\ k\end{array}\right) x^{k}$ and so conclude the following.

Proposition 3.1.5

$$
C_{\ell}(x)=\frac{1}{(1-x)^{\ell-1}}
$$




\subsection{Decomposing $\ell$-partitions}

We now present our decomposition of $\ell$-partitions. Let $\mu=\left(\mu_{1}, \ldots, \mu_{s}\right)$ be any $\ell$-core where $\mu_{1}-\mu_{2} \neq$ $\ell-1$. For an $r \geq 0$ we form a new $\ell$-core $\nu=\left(\nu_{1}, \ldots \nu_{r}, \nu_{r+1}, \ldots, \nu_{r+s}\right)$ by attaching $r$ rows above $\mu$ so that $\nu_{r}=\mu_{1}+\ell-1, \nu_{r-1}=\mu_{1}+2(\ell-1), \ldots \nu_{1}=\mu_{1}+r(\ell-1)$ and $\nu_{r+i}=\mu_{i}$ for $i=1,2, \ldots, s$. Here $r$ may be zero, in which case $\mu=\nu$. It can be shown that $\nu$ is an $\ell$-core. Fix a partition $\kappa=\left(\kappa_{1}, \ldots, \kappa_{r+1}\right)$ with at most $(r+1)$ parts. Then a new partition $\lambda$ is obtained from $\nu$ by adding $\kappa_{i}$ horizontal $\ell$-rim hooks to row $i$ for every $i \in\{1, \ldots, r+1\}$. In other words $\lambda_{i}=\nu_{i}+\ell \kappa_{i}$ for $i \in\{1,2, \ldots, r+1\}$ and $\lambda_{i}=\nu_{i}$ for $i>r+1$.

From now on, when we associate $\lambda$ with the triple $(\mu, r, \kappa)$, we will think of $\mu \subset \lambda$ as embedded in the rows below the $r$ th row in $\lambda$. We introduce the notation $\lambda \approx(\mu, r, \kappa)$ for this decomposition.

Theorem 3.2.1 Let $\mu, r$ and $\kappa$ be as above. Then $\lambda \approx(\mu, r, \kappa)$ is an $\ell$-partition. Conversely, every $\ell$-partition corresponds uniquely to a triple $(\mu, r, \kappa)$.

Example 3.2.2 For $\ell=3, \mu=(2,1,1)$ is a 3-core with $\mu_{1}-\mu_{2} \neq 2$. We may add three rows $(r=3)$ to it to obtain $\nu=(8,6,4,2,1,1)$, which is still a 3-core. Now we may add three horizontal $\ell$-rim hooks to the first row, three to the second, one to the third and one to the fourth $(\kappa=(3,3,1,1))$ to obtain the partition $\lambda=(17,15,7,5,1,1)$, which is a 3-partition.

Remark 3.2.3 In the proofs of Theorems 4.3.1 and 4.3.3 we will prove that a partition is an $\ell$-partition by giving its decomposition into $(\mu, r, \kappa)$.

\subsection{Counting $\ell$-partitions}

We derive a closed formula for our generating function $B_{\ell}$ by using our $\ell$-partition decomposition described above. First we note that if

$$
C_{\ell}(x)=\sum_{\mu \in \mathcal{C}_{\ell}} x^{\mu_{1}} \quad \text { then } \quad x^{\ell-1} C_{\ell}(x)=\sum_{\mu \in \mathcal{C}_{\ell}: \mu_{1}-\mu_{2}=\ell-1} x^{\mu_{1}} .
$$

Therefore, $\sum_{\mu \in \mathcal{C}_{\ell}: \mu_{1}-\mu_{2} \neq \ell-1} x^{\mu_{1}}=\left(1-x^{\ell-1}\right) C_{\ell}(x)$. Hence the generating function for all cores $\mu$ with $\mu_{1}-\mu_{2} \neq \ell-1$ is $\frac{1-x^{\ell-1}}{(1-x)^{\ell-1}}$.

We are now ready to prove our closed formula for $B_{\ell}$. First, recall that $B_{\ell}(x)=\sum b_{n}^{\ell} x^{n}$ where $b_{n}^{\ell}=$ $\left\{\lambda \in \mathcal{L}_{\ell}: \lambda_{1}=n\right\}$.

Theorem 3.3.1

$$
B_{\ell}(x)=\frac{1-x^{\ell-1}}{(1-x)^{\ell-1}\left(1-x^{\ell-1}-x^{\ell}\right)} .
$$

Proof: We follow our construction of $\ell$-partitions from Section 3.2. Note that if $\lambda \approx(\mu, r, \kappa)$, then the first part of $\lambda$ is $\mu_{1}+\ell \kappa_{1}+r(\ell-1)$. Hence $\lambda$ contributes $x^{\mu_{1}+\ell \kappa_{1}+r(\ell-1)}$ to $B_{\ell}$.

From here we apply a simple counting argument to conclude our theorem. 


\section{The Crystal of the Basic Representation of $\widehat{\mathfrak{s l}_{\ell}}$}

There is a crystal graph structure on the set of $\ell$-regular partitions. In this section we examine the properties of $\ell$-partitions in this crystal graph. In particular, just as one can obtain $\ell$-cores by following an $i$-string from another $\ell$-core, one can obtain $\ell$-partitions by following an $i$-string from another $\ell$-partition. However, more $\ell$-partitions exist. Sometimes $\ell$-partitions live one position before the end of an $i$-string (but nowhere else). In particular, there is not a nice interpretation in terms of the braid group orbits of nodes. In Theorem 4.3.3 we characterize (combinatorially) where this happens.

\subsection{Description of crystal}

We will assume some familiarity with the theory of crystals (see [6]), and their relationship to the representation theory of the finite Hecke Algebra (see [3] or [7]). We will look at the crystal $B\left(\Lambda_{0}\right)$ of the irreducible highest weight module $V\left(\Lambda_{0}\right)$ of the affine Lie algebra $\widehat{\mathfrak{s l}}_{\ell}$ (also called the basic representation of $\left.\widehat{\mathfrak{s l}_{\ell}}\right)$. The set of nodes of $B\left(\Lambda_{0}\right)$ will be denoted $B:=\{\lambda \in \mathcal{P}: \lambda$ is $\ell$-regular $\}$. We will describe the arrows of $B\left(\Lambda_{0}\right)$ below. This description is originally due to Misra and Miwa (see [10]).

We now view the Young diagram for $\lambda$ as a set of boxes, with their corresponding residues $b-a \bmod \ell$ written into the box in row $a$ and column $b$. A box $x$ in $\lambda$ is said to be a removable $i$-box if it has residue $i$ and after removing $x$ from $\lambda$ the remaining diagram is still a partition. A box $y$ not in $\lambda$ is an addable $i$-box if it has residue $i$ and adding $y$ to $\lambda$ yields a partition.

For a fixed $i,(0 \leq i<\ell)$, we place - in each removable $i$-box and + in each addable $i$-box. The $i$-signature of $\lambda$ is the word of + and -'s in the diagram for $\lambda$, read from bottom left to top right. The reduced $i$-signature is the word obtained after repeatedly removing from the $i$-signature all adjacent pairs -+ . The reduced $i$-signature is of the form $+\cdots+++---\cdots-$. The boxes corresponding to - 's in the reduced $i$-signature are called normal $i$-boxes, and the boxes corresponding to + 's are called conormal $i$-boxes. $\varepsilon_{i}(\lambda)$ is defined to be the number of normal $i$-boxes of $\lambda$, and $\varphi_{i}(\lambda)$ is defined to be the number of conormal $i$-boxes. If a leftmost - exists in the reduced $i$-signature, the box corresponding to said - is called the good $i$-box of $\lambda$. If a rightmost + exists in the reduced $i$-signature, the box corresponding to said + is called the cogood $i$-box. All of these definitions can be found in Kleshchev's book [7].

We recall the action of the crystal operators on $B$. The crystal operator $\widetilde{e}_{i}: B \stackrel{i}{\rightarrow} B \cup\{0\}$ assigns to a partition $\lambda$ the partition $\widetilde{e}_{i}(\lambda)=\lambda \backslash x$, where $x$ is the good $i$-box of $\lambda$. If no such box exists, then $\widetilde{e}_{i}(\lambda)=0$. It is clear that $\varepsilon_{i}(\lambda)=\max \left\{k: \widetilde{e}_{i}^{k} \lambda \neq 0\right\}$.

Similarly, $\widetilde{f}_{i}: B \stackrel{i}{\rightarrow} B \cup\{0\}$ is the operator which assigns to a partition $\lambda$ the partition $\widetilde{f}_{i}(\lambda)=\lambda \cup x$, where $x$ is the $\operatorname{cogood} i$-box of $\lambda$. If no such box exists, then $\widetilde{f}_{i}(\lambda)=0$. It is clear that $\varphi_{i}(\lambda)=\max \{k$ : $\left.\widetilde{f}_{i}^{k} \lambda \neq 0\right\}$.

For $i$ in $\mathbb{Z} / \ell \mathbb{Z}$, we write $\lambda \stackrel{i}{\rightarrow} \mu$ to stand for $\widetilde{f}_{i} \lambda=\mu$. We say that there is an $i$-arrow from $\lambda$ to $\mu$. Note that $\lambda \stackrel{i}{\rightarrow} \mu$ if and only if $\widetilde{e}_{i} \mu=\lambda$. A maximal chain of consecutive $i$-arrows is called an $i$-string. We note that the empty partition $\emptyset$ is the unique highest weight node of the crystal. For a picture of the first few levels of this crystal graph, see [8] for the cases $\ell=2$ and 3.

Example 4.1.1 Let $\lambda=(8,5,4,1)$ and $\ell=3$. Then $\widetilde{e}_{1}(8,5,4,1)=(7,5,4,1)$ and $\widetilde{f}_{1}(8,5,4,1)=$ $(8,5,4,2)$. Also, $\widetilde{e}_{1}^{2}(8,5,4,1)=0$ and $\widetilde{f}_{1}^{2}(8,5,4,1)=0$. The sequence $(7,5,4,1) \stackrel{1}{\rightarrow}(8,5,4,1) \stackrel{1}{\rightarrow}$ $(8,5,4,2)$ is a 1 -string of length 3. 


\subsection{Crystal operators and $\ell$-partitions}

We first recall some well-known facts about the behavior of $\ell$-cores in this crystal graph $B\left(\Lambda_{0}\right)($ See [6] for more details). There is an action of the affine Weyl group $\widetilde{S}_{\ell}$ on the crystal such that the simple reflection $s_{i}$ reflects each $i$-string. The $\ell$-cores are exactly the $\widetilde{S}_{\ell}$-orbit of $\emptyset$, the highest weight node. This implies the following Proposition.

Proposition 4.2.1 If $\mu$ is an $\ell$-core and $\varepsilon_{i}(\mu) \neq 0$ then $\varphi_{i}(\mu)=0$ and $\widetilde{e}_{i}^{\varepsilon_{i}(\mu)} \mu$ is again an $\ell$-core. Furthermore, all $\widetilde{e}_{i}^{k} \mu$ for $0<k<\varepsilon_{i}(\mu)$ are not $\ell$-cores. Similarly, if $\varphi_{i}(\mu) \neq 0$ then $\varepsilon_{i}(\mu)=0$ and $\widetilde{f}_{i}^{\varphi_{i}(\mu)} \mu$ is an $\ell$-core but $\widetilde{f}_{i}^{k} \mu$ is not for $0<k<\varphi_{i}(\mu)$.

In this paper, given an $\ell$-partition $\lambda$, we will determine when $\widetilde{f}_{i}^{k} \lambda$ and $\widetilde{e}_{i}^{k} \lambda$ are also $\ell$-partitions.

The following remarks will help us in the proofs of the upcoming Theorems 4.3.1, 4.3.2 and 4.3.3.

Remark 4.2.2 Suppose $\lambda$ is a partition. Consider its Young diagram. If any $\ell$-rim hook has an upper rightmost box of residue $i$, then the lower leftmost box has residue $i+1 \bmod \ell$. Conversely, a hook length $h_{(a, b)}^{\lambda}$ is divisible by $\ell$ if and only if there is an $i$ so that the rightmost box of row a has residue $i$, and the lowest box of column $b$ has residue $i+1 \bmod \ell$.

In Lemma 4.2.4 we will generalize Proposition 4.2.3 to $\ell$-partitions. The proofs are omitted in the interest of space.

Proposition 4.2.3 Let $\lambda$ be an $\ell$-core, and suppose $0 \leq i<\ell$. Then the $i$-signature for $\lambda$ is the same as the reduced $i$-signature.

Lemma 4.2.4 Let $\lambda$ be an $\ell$-partition, and suppose $0 \leq i<\ell$. Then the $i$-signature for $\lambda$ is the same as the reduced $i$-signature.

Remark 4.2.5 As a consequence of Lemma 4.2.4, the action of the operators $\widetilde{e}_{i}$ and $\widetilde{f}_{i}$ are simplified in the case of $\ell$-partitions. Now applying successive $\widetilde{f}_{i}$ 's to $\lambda$ corresponds to adding all addable boxes of residue i from right to left. Similarly, applying successive $\widetilde{e}_{i}$ 's to $\lambda$ corresponds to removing all removable boxes of residue i from left to right.

In the following Theorems 4.3.1, 4.3.2 and 4.3.3, we implicitly use Remark 4.2.2 to determine when a hook length is divisible by $\ell$, and Remark 4.2 .5 when applying $\widetilde{e}_{i}$ and $\widetilde{f}_{i}$ to $\lambda$. Unless it is unclear from the context, for the rest of the paper $\varphi=\varphi_{i}(\lambda)$ and $\varepsilon=\varepsilon_{i}(\lambda)$.

Remark 4.2.6 Suppose $\lambda \approx(\mu, r, \kappa)$. When viewing $\mu$ embedded in $\lambda$, we note that if a box $(a, b) \in \mu \subset$ $\lambda$ has residue $i \bmod \ell$ in $\lambda$, then it has residue $i-r \bmod \ell$ in $\mu$.

For a partition $\lambda=\left(\lambda_{1}, \lambda_{2}, \ldots, \lambda_{k}\right)$ of length $k$, we define $\bar{\lambda}=\left(\lambda_{2}, \ldots, \lambda_{k}\right), \hat{\lambda}=\left(\lambda_{1}, \lambda_{1}, \lambda_{2}, \lambda_{3}, \ldots, \lambda_{k}\right)$ and $\lambda+1=\left(\lambda_{1}+1, \lambda_{2}+1, \ldots, \lambda_{k}+1\right)$. Note that if $\lambda$ is an $\ell$-core, then so is $\bar{\lambda}$.

\section{$4.3 \ell$-partitions in the crystal $B\left(\Lambda_{0}\right)$}

Theorem 4.3.1 Suppose that $\lambda$ is an $\ell$-partition and $0 \leq i<\ell$. Then

1. $\tilde{f}_{i}^{\varphi} \lambda$ is an $\ell$-partition,

2. $\widetilde{e}_{i}^{\varepsilon} \lambda$ is an $\ell$-partition. 
Proof: We will prove only (1), as (2) is similar. Recall all addable $i$-boxes of $\lambda$ are conormal by Lemma 4.2.4. The proof of (1) relies on the decomposition of the $\ell$-partition as in Section 3.2. Let $\lambda \approx(\mu, r, \kappa)$. We break the proof of (1) into three cases:

(a) If the first row of $\mu$ embedded in $\lambda$ does not have an addable $i$-box then we cannot add an $i$-box to the first $r+1$ rows of $\lambda$. Hence $\varphi=\varphi_{i-r}(\mu)$. Therefore the decomposition of $\widetilde{f}_{i}^{\varphi} \lambda$ has the same $r$ and $\kappa$ as $\lambda$, and $\mu$ will be replaced by $\widetilde{f}_{i-r}^{\varphi} \mu$, which is still a core by Proposition 4.2.3. Hence $\widetilde{f}_{i}^{\varphi} \lambda \approx\left(\widetilde{f}_{i-r}^{\varphi} \mu, r, \kappa\right)$.

(b) If the first row of $\mu$ embedded in $\lambda$ does have an addable $i$-box and $\mu_{1}-\mu_{2}<\ell-2$, then the first $r+1$ rows of $\lambda$ have addable $i$-boxes. Also some rows of $\mu$ will have addable $i$-boxes. $\widetilde{f}_{i}^{\varphi}$ adds an $i$-box to the first $r$ rows of $\lambda$, plus adds to any addable $i$-boxes in the core $\mu$. Note that $\varphi_{i-r}(\mu)=\varphi-r$. Since $\mu_{1}-\mu_{2}<\ell-2$, the first and second rows of $\widetilde{f}_{i-r}^{\varphi-r} \mu$ differ by at most $\ell-2$. Therefore $\widetilde{f}_{i}^{\varphi} \lambda \approx$ $\left(\widetilde{f}_{i-r}^{\varphi-r} \mu, r, \kappa\right)$.

(c) If the first row of $\mu$ embedded in $\lambda$ does have an addable $i$-box and $\mu_{1}-\mu_{2}=\ell-2$, then $\widetilde{f}_{i}^{\varphi}$ will add the addable $i$-box in the $r+1^{\text {st }}$ row (i.e. the first row of $\mu$ ). Since the $(r+2)^{n d}$ row does not have an addable $i$-box, we know that the $(r+1)^{s t}$ and $(r+2)^{n d}$ rows of $\widetilde{f}_{i}^{\varphi}(\lambda)$ differ by $\ell-1$. Therefore $\tilde{f}_{i}^{\varphi} \lambda \approx\left(\overline{\widetilde{f}_{i-r}^{\varphi-r} \mu}, r+1, \kappa\right)$ is an $\ell$-partition.

Theorem 4.3.2 Suppose that $\lambda$ is an $\ell$-partition. Then

1. $\widetilde{f}_{i}^{k} \lambda$ is not an $\ell$-partition for $0<k<\varphi-1$,

2. $\widetilde{e}_{i}^{k} \lambda$ is not an $\ell$-partition for $1<k<\varepsilon$.

\section{Proof:}

If $0<k<\varphi-1$ then there are at least two conormal $i$-boxes in $\widetilde{f}_{i}^{k} \lambda$ and at least one normal $i$-box, to the right of the two conormal $i$-boxes by Lemma 4.2.4. Label the conormal boxes $n_{1}$ and $n_{2}$, with $n_{1}$ to the left of $n_{2}$. Call the normal box $n_{3}$. Then the hook length in the column of $n_{1}$ and row of $n_{3}$ is a multiple of $\ell$, but the hook length in the column of $n_{1}$ and row of $n_{2}$ is not a multiple of $\ell$ by Remark 4.2.2. By Theorem 2.1.6, $\widetilde{f}_{i}^{k} \lambda$ is not an $\ell$-partition. The proof of (2) is similar to (1).

The above theorems told us the position of an $\ell$-partition relative to the $i$-string which it sits on in the crystal $B\left(\Lambda_{0}\right)$. If an $\ell$-partition occurs on an $i$-string, then both ends of the $i$-string are also an $\ell$-partitions. Furthermore, the only places $\ell$-partitions can occur are at the ends of $i$-strings or possibly one position before the final node. The next theorem describes when this latter case occurs.

Theorem 4.3.3 Suppose that $\lambda \approx(\mu, r, \kappa)$ is an $\ell$-partition. Then

1. If $\varphi>1$ then $\widetilde{f}_{i}^{\varphi-1} \lambda$ is an $\ell$-partition if and only if

$(\dagger) \quad \kappa_{r+1}=0$, the first row of $\lambda$ has a conormal $i$-box, and $\varphi=r+1$.

2. If $\varepsilon>1$ then $\widetilde{e}_{i} \lambda$ is an $\ell$-partition if and only if

( $\ddagger)$ the first row of $\lambda$ has a conormal $(i+1)$-box and either $\varepsilon=r$ and $\kappa_{r}=0$, or $\varepsilon=r+1$ and $\kappa_{r+1}=0$. 
Proof: We first prove (1) and then derive (2) from (1).

If $\lambda$ satisfies condition $(\dagger)$ then $\widetilde{f}_{i}^{\varphi-1} \lambda \approx(\widehat{\mu}, r-1, \kappa+1)$, so it is an $\ell$-partition.

Conversely:

(a) If the first part of $\lambda$ has a conormal $j$-box, with $j \neq i$, call this box $n_{1}$. If $j=i+1$ then the box $\left(r+1, \lambda_{r+1}\right)$ has residue $i$. If an addable $i$-box exists, say at $(a, b)$, it must be below the first $r+1$ rows. But then the hook length $h_{(r+1, b)}^{\mu}$ is divisible by $\ell$. This implies that $\mu$ is not a core. So we assume $j \neq i+1$. Then $\tilde{f}_{i}^{\varphi-1} \lambda$ has at least one normal $i$-box $n_{2}$ and exactly one conormal $i$-box $n_{3}$ with $n_{3}$ left of $n_{2}$ left of $n_{1}$. The hook length of the box in the column of $n_{3}$ and row $n_{2}$ is divisible by $\ell$, but the hook length in the box of column $n_{3}$ and row $n_{1}$ is not (by Remark 4.2.2). By Theorem 2.1.6, $\widetilde{f}_{i}^{\varphi-1} \lambda$ is not an $\ell$-partition.

(b) By (a), we can assume that the first row has a conormal $i$-box. If $\varphi \neq r+1$ then row $r+2$ of $\tilde{f}_{i}^{\varphi-1} \lambda$ will end in a $j$-box, for some $j \neq i$. Call this box $n_{1}$. Also let $n_{2}$ be any normal $i$-box in $\tilde{f}_{i}^{\varphi-1} \lambda$ and $n_{3}$ be the unique conormal $i$-box. Then the box in the row of $n_{1}$ and column of $n_{3}$ has a hook length which is not divisible by $\ell$, but the box in the row of $n_{2}$ and column of $n_{3}$ has a hook length which is (by Remark 4.2.2). By Theorem 2.1.6, $\widetilde{f}_{i}^{\varphi-1} \lambda$ is not an $\ell$-partition.

(c) Suppose $\kappa_{r+1} \neq 0$. By $(a)$ and $(b)$, we can assume that $\varphi=r+1$ and that the first row of $\lambda$ has a conormal $i$-box. Then the difference between $\lambda$ and $\tilde{f}_{i}^{\varphi-1} \lambda=\widetilde{f}_{i}^{r} \lambda$ is an added box in each of the first $r$ rows. Remove $\kappa_{r}-\kappa_{r+1}+1$ horizontal $\ell$-rim hooks from row $r$ of $\tilde{f}_{i}^{\varphi-1} \lambda$. Call the remaining partition $\nu$. Then $\nu_{r}=\nu_{r+1}=\mu_{1}+\ell \kappa_{r+1}$. Hence a removable non-horizontal $\ell$-rim hook exists in $\nu$ taking the rightmost box from row $r$ with the rightmost $\ell-1$ boxes from row $r+1$. Thus $\widetilde{f}_{i}^{\varphi-1}(\lambda)$ is not an $\ell$-partition.

To prove (2), we note that by Theorem (4.3.2) that if $\varphi \neq 0$ and $\varepsilon>1$ then $\widetilde{e}_{i} \lambda=\widetilde{e}_{i}^{2} \widetilde{f}_{i} \lambda$ cannot be an $\ell$-partition. Hence we only consider $\lambda$ so that $\varphi_{i}(\lambda)=0$. But then $\widetilde{f}_{i}^{\varphi_{i}}\left(\widetilde{e}_{i}^{\varepsilon}(\lambda)\right)-1 \widetilde{e}_{i}^{\varepsilon} \lambda=\widetilde{f}_{i}^{\varepsilon-1} \widetilde{e}_{i}^{\varepsilon} \lambda=\widetilde{e}_{i} \lambda$. From this observation, it is enough to show that $\lambda$ satisfies ( $\ddagger$ ) if and only if $\widetilde{e}_{i}^{\varepsilon} \lambda$ satisfies (†). The proof of this follows a similar line as the above proofs, so it will be left to the reader.

Example 4.3.4 Fix $\ell=3$. Let $\lambda=(9,4,2,1,1) \approx((2,1,1), 2,(1))$.

Here $\varphi_{0}(\lambda)=3$. $\widetilde{f}_{0} \lambda=(10,4,2,1,1)$ is not a 3-partition, but $\widetilde{f}_{0}^{2} \lambda=(10,5,2,1,1) \approx((2,2,1,1), 1,(2,1))$ and $\widetilde{f}_{0}^{3} \lambda=(10,5,3,1,1) \approx((1,1), 3,(1))$ are 3 -partitions.

\section{Further Work}

Recent results of Fayers ([2]) and Lyle ([9]) have given combinatorial conditions which characterize partitions $\lambda$ so that the corresponding Specht modules $S^{\lambda}$ of $H_{n}(q)$ stay irreducible when $q$ is a primitive $\ell$ th root of unity, without the condition that the $\lambda$ be $\ell$-regular $(\ell>2)$. All of the above work can be generalized to this setting (considering vertical and horizontal $\ell$-rim hooks and a similar decomposition).

\section{References}

[1] R. Dipper and G. James, Representations of Hecke algebras of general linear groups, Proc. LMS (3), 52 (1986), 20-52

[2] M. Fayers, Irreducible Specht modules for Hecke Algebras of Type A, Adv. Math. 193 (2005) 438452 
[3] I. Grojnowski, Affine $\widehat{\mathfrak{s l}_{\mathfrak{p}}}$ controls the representation theory of the symmetric group and related Hecke algebras, ArXiv Mathematics e-prints, math/9907129

[4] G. D. James and A. Kerber, The Representation Theory of the Symmetric Group, Encyclopedia of Mathematics, 16, 1981.

[5] G.D. James and A. Mathas, A q-analogue of the Jantzen-Schaper theorem, Proc. Lond. Math. Soc., 74 (1997), 241-274.

[6] M. Kashiwara, On crystal bases, in Representations of groups (Banff 1994), CMS Conf. Proc. 16 (1995), 155-197.

[7] A. Kleshchev, Linear and Projective Representations of Symmetric Groups, Cambridge Tracts in Mathematics 163.

[8] A. Lascoux, B. Leclerc, and J.-Y. Thibon, Hecke algebras at roots of unity and crystal bases of quantum affine algebras, Comm. Math. Phys. 181 (1996), 205-263.

[9] S. Lyle, Some q-analogues of the Carter-Payne Theorem, J. Reine Angew. Math., to appear

[10] K.C. Misra and T. Miwa, Crystal base for the basic representation of $U_{q}\left(\mathfrak{s l}_{n}\right)$, Comm. Math. Phys. 134 (1990), 79-88. 